N. Cabibbo in his two page long paper proposed a solution to both problems. He defined an octet of unit currents $J_{\mu}$ with vector and axial vector components to describe strangeness changing and strangeness non-changing weak decays. The weights for the two components were expressed as $\sin$ and $\cos$ of an angle $\theta$, the Cabibbo angle. By comparing $\mathrm{K}^{+} \rightarrow \mu^{+} v$ with $\pi^{+} \rightarrow \mu^{+} v$ he obtained a value of $\theta$ $=0.26$ which is very close to the number given in the most recent edition of the particle data tables. This approach applied to hyperon decays led to predictions of branching ratios which agree within errors with today's measurements. The ansatz of current mixing explains at the same time the difference in the vector coupling constants measured in nuclear $\beta$ decay and $\mu$ decay. Thus three pending problems were solved at once.

Only one year later, in 1964, Gell-Mann and Zweig introduced quarks to physics and Gell-Mann rewrote Cabibbo's octet of currents in terms of quark wave functions

$$
J_{\mu}=u \gamma_{\mu}\left(1+\gamma_{5}\right)(d \cos \theta+s \sin \theta) \text {. }
$$

In 1970, six years later, S.L. Glashow, J. Iliopoulos and L. Maiani (GIM) introduced two quark doublets with pure charged $2 / 3$ state and mixed charged $-1 / 3$ states in order to correct some shortcomings of the weak interaction theory. The weak hadronic current then reads as

$$
J_{\mu}=(u, c)\left(\begin{array}{c}
\cos \theta \sin \theta \\
\sin \theta \cos \theta
\end{array}\right) \gamma_{\mu}\left(1+\gamma_{5}\right)\left(\begin{array}{l}
d \\
s
\end{array}\right)
$$

The last step brings us to the present status of the quark mixing scheme with three doublets and the incorporation of $\mathrm{CP}$ violation through a non-trivial phase in the Cabibbo-Kobayashi-Maskawa matrix:

$$
\begin{aligned}
& a_{1}-\left(\begin{array}{c}
u \\
\alpha_{11} d+\alpha_{12} s+\alpha_{13} b
\end{array}\right) \\
& a_{2}-\left(\begin{array}{c}
\alpha_{21} d+\alpha_{22} s+\alpha_{23} b
\end{array}\right) \\
& a_{3}-\left(\begin{array}{c}
\alpha_{31} d+\alpha_{32} s+\alpha_{33} b
\end{array}\right)
\end{aligned}
$$

with the corresponding weak current

$$
J_{\mu}=(u, c, t) M \gamma_{\mu}\left(1+\gamma_{5}\right)\left(\begin{array}{l}
d \\
s \\
b
\end{array}\right)
$$

where $M$ is the Cabibbo-Kobayashi-Maskawa matrix. Considerable experimental effort is being devoted at present to determining the elements of the matrix and to understanding the origin of CP violation.

The EPS High Energy Physics Prize is sponsored by industry. The following firms have contributed:

Cray Research France (France)

Digital Equipment Corporation Europe

(Switzerland)

Interatom (Germany)

Le Croy (USA)

Philips (The Netherlands)

Siemens AG (Germany)

Thomson Tubes Electroniques (France)

\section{W. Bartel, DESY, Hamburg}

\title{
The 17 keV Neutrino
}

\section{Quest Continues With Improved Techniques}

Confirming the existence of a $17 \mathrm{keV}$ neutrino would represent the first evidence for a physics beyond the standard model with a breakdown of the electroweak theory predicting massless neutrinos (extended models propose Dirac or Majorana mass terms depending on the symmetry of the underlying theory). Evidence for the $17 \mathrm{keV}$ neutrino was first reported in 1985 by John Simpson in the $\beta$ decay of ${ }^{3} \mathrm{H}$ implanted in a Si-Li detector. Six experiments in 1985-89 looking for distortions of the $\beta$ spectra of ${ }^{35} \mathrm{~S}$ and ${ }^{63} \mathrm{Ni}$ owing to the emission of the $17 \mathrm{keV}$ neutrino then gave negative results.

In 1991, Simpson and Hime (working with ${ }^{3} \mathrm{H}$ and ${ }^{35} \mathrm{~S}$ ) and Hime and Jelley ( ${ }^{14} \mathrm{~S}$ with a $\mathrm{Si}$ - $\mathrm{Li}$ detector) then obtained another positive result, as did groups at Berkeley $\left({ }^{14} \mathrm{C}\right)$ and Zagreb $\left({ }^{7} \mathrm{Ge}\right)$ also using semiconductor detectors. G.G. Ross mentioned a preliminary report of another positive result - this time for ${ }^{63} \mathrm{Ni}$ - at the 1991 LP-HEP Conference.

However, Becker et al. at Caltech using a ${ }^{35} \mathrm{~S}$ source and a double focussing spectrometer reported negative results. Additional negative results were presented at the LP-HEP Conference by Bahran and Kalbfleisch who measured $\beta$ decay in ${ }^{3} \mathrm{H}$ with an apparatus constructed of hydrogen-free material (to reduce distortion of the $\beta$ spectrum by adsorption from the walls). Curve fitting spectra showed that a $<0.4 \%$ admixture of the $17 \mathrm{keV}$ neutrino could be excluded with a $99 \%$ confidence limit.

\section{Physics Heads Meet}

Some so-called "mainstream" fields of physics (fission, high energy, synchrotron radiation, nuclear) have well established organizational structures while others (neutron scattering, high magnetic fields) are developing arrangements. Dr. K.H. Chang, Director of the Dutch physics research organization FOM, speaking at the meeting of the EPS Associate Members in Cracow last month, stressed how the historical "internationalism" of physics as well as political pressure for europeanization call for new networks and large facilities. National funding agencies find planning within this framework increasingly difficult owing to reductions in long-term structural investments being offset by special short-term funding. Dr. Chang reported on a first meeting, held last April in the UK, of a group with the provisional title CEPCRC (Chairman of the European Physics Committees

\section{W. Czaja (Ed.)}

Synchrotron Radiation:

Selected Experiments in

Condensed Matter Physics

In this volume, 10 contributions describe selected experiments in condensed matter physics with synchrotron radiation, the subject matter at an international workshop held at the Centro Stefano Franscini in Ascona, Switzerland, in July 1990. The experiments concerned magnetic properties, electrical properties of clusters, liquid metals and magnetic semiconductors, interface problems (Schottky barriers), etc. They demonstrate the enormous impact and challenge which lies in the use of a synchrotron light source in many areas of solid state physics. In another paper, applications of the new light source are discussed in relation with the determination of crystal structures. Finally, information on the properties and experimental possibilities of the new synchrotron in Trieste are given. This installation is one of the three new third generation synchrotrons now under construction and promises once again to enlarge the number of possible applications. of the Research Councils) where physics division heads of national funding agencies considered mechanisms to stimulate multinational collaboration. The CEPCRC's next meeting will be hosted by Germany.

\section{Quantum Electronics \& Optics Division}

The 1991 EPS Council in Zurich recommended that the Quantum Electronics Division (OED) consult members to see if the Division's name should be changed to reflect wider interests in meeting the needs of members belonging to the former Optics Division. Professor Peter Knight, Chairman of the QED Board, reports that, on the Board's recommendation, the Division Business Meeting held in September in Edinburgh voted unanimously to adopt the name "The Quantum Electronics and Optics Division".
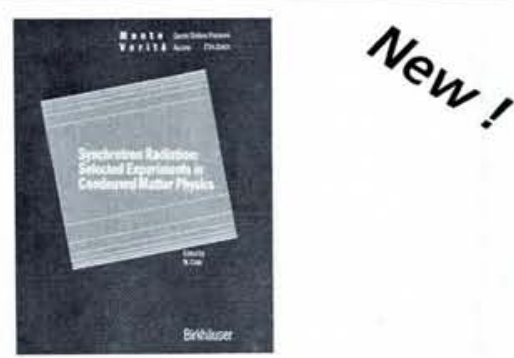

1991. Approx. 168 pages. Hardcover SFr. 58.- I DM 68. ISBN 3-7643-2594-1

Please send your orders to: Birkhäuser Verlag AG, P.O. Box 133 $\mathrm{CH}-4010$ Basel / Switzerland

Orders originating from the USA or Canada please send to: Birkhăuser Boston, Inc., Clo Springer Verlag New York, Inc. 44 Hartz Way, Secaucus NJ, 07096-2491, USA

\section{Birkhäuser}

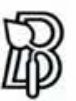

Birkhäuser Verlag AG Basel-Boston-Berlin 